\title{
UCRL-JC-116148
}

PREPRINT

\section{Automated Fiber Pigtailing Technology}

\author{
O.T. Strand, M.E. Lowry, S.Y. Lu, D.C. Nelson, D.J. Nikkel, \\ M.D. Pocha, K.D. Young
}

This paper was prepared for submittal to the 44th Electronic Components and Technology Conference Washington, D.C. May 1-4, 1994

February 1994

This is a preprint of a paper intended for publication in a journal or proceedings. Since changes may be made before publication, this preprint is made available with the understanding that it will not be cited or reproduced without the permission of the author. 


\section{DISCLAIMER}

This document was prepared as an account of work sponsored by an agency of the United States Government. Nelther the United States Government nor the University of Callfornia nor any of their employees, makes any warranty, express or implied, or assumes any legal liabillity or responsibility for the accuracy, completeness, or usefulness of any information, apparatus, product, or process disclosed, or represents that its use would not infringe privately owned rights. Reference herein to any spectinc commercial products, process, or service by trade name, trademark, manufacturer, or otherwise, does not aecessarily constilute or imply its endorsement, recommendation, or favoring by the United States Government or the University of California. The views and opinions of authors expressed herein do not necessarily state or reflect those of the United States Governmeat or the University of California, and shall not be used for advertising of product endorsement purposes. 


\title{
Automated Fiber Pigtailing Technology
}

\author{
O.T. Strand, M.E. Lowry, S.Y. Lu, D.C. Nelson, D.J. Nikkel, M.D. Pocha, K.D. Young \\ Lawrence Livermore National Laboratory \\ Livermore, CA 94550
}

Abstract - The high cost of optoelectronic (OE) devices is due mainly to the labor-intensive packaging process. Manually pigtailing such devices as single-mode laser diodes and modulators is very time consuming with poor quality control. The Photonics Program and the Engineering Research Division at LLNL are addressing several issues associated with automatically packaging $\mathrm{OE}$ devices. A fully automated system must include high-precision fiber alignment, fiber attachment techniques, in-situ quality control, and parts handling and feeding. This paper will present on-going work at LLNL in the areas of autornated fiber alignment and fiber attachment. For the fiber alignment, we are building an automated fiber pigtailing machine (AFPM) which combines computer vision and object recognition algorithms with active feedback to perform sub-micron alignments of single-mode fibers to modulators and laser diodes. We expect to perform sub-micron alignments in less than five minutes with this technology. For fiber attachment, we are building various geometries of silicon microbenches which include onboard heaters to solder metal-coated fibers and other components in place; these designs are completely compatible with an automated process of $O E$ packaging. We have manually attached a laser diode, a thermistor, and a thermo-electric heater to one of our microbenches in less than 15 minutes using the on-board heaters for solder reflow; an automated process could perform this same exercise in only a few minutes. Automated packaging techniques such as these will help lower the costs of $\mathrm{OE}$ devices.

\section{INTRODUCTION}

\section{$O E$ devices cost too much}

At present, the cost of optoelectronic devices is dominated by the effort required to package those devices into an integrated system. Components such as laser diodes and modulators, designed for high-performance applications, are single-mode devices; they must be connected together using optical fibers or other type of waveguide with sub-micron alignment accuracies. Presently, OE packaging is usually performed by highly skilled technicians looking through microscopes and manually adjusting sub-micron stages. Once the alignment is correct, the components must be held in place using epoxy, solder, or other attachment technique in such a manner that none of the components move during the attachment process. This labor-intensive process results in only a few packages being produced per day by each technician. The packaging costs are by far the highest fraction of the total cost of an assembled OE package. The consequences of this low-volume labor-intensive process of packaging $\mathrm{OE}$ devices are readily apparent. The costs are too high to allow the advantages of fiber optics to penetrate such markets as on-chip interconnects, interboard connections in computers, local area networks, or fiber to the home.

\section{OE packaging must be automated}

At LLNL, we believe that the pack-

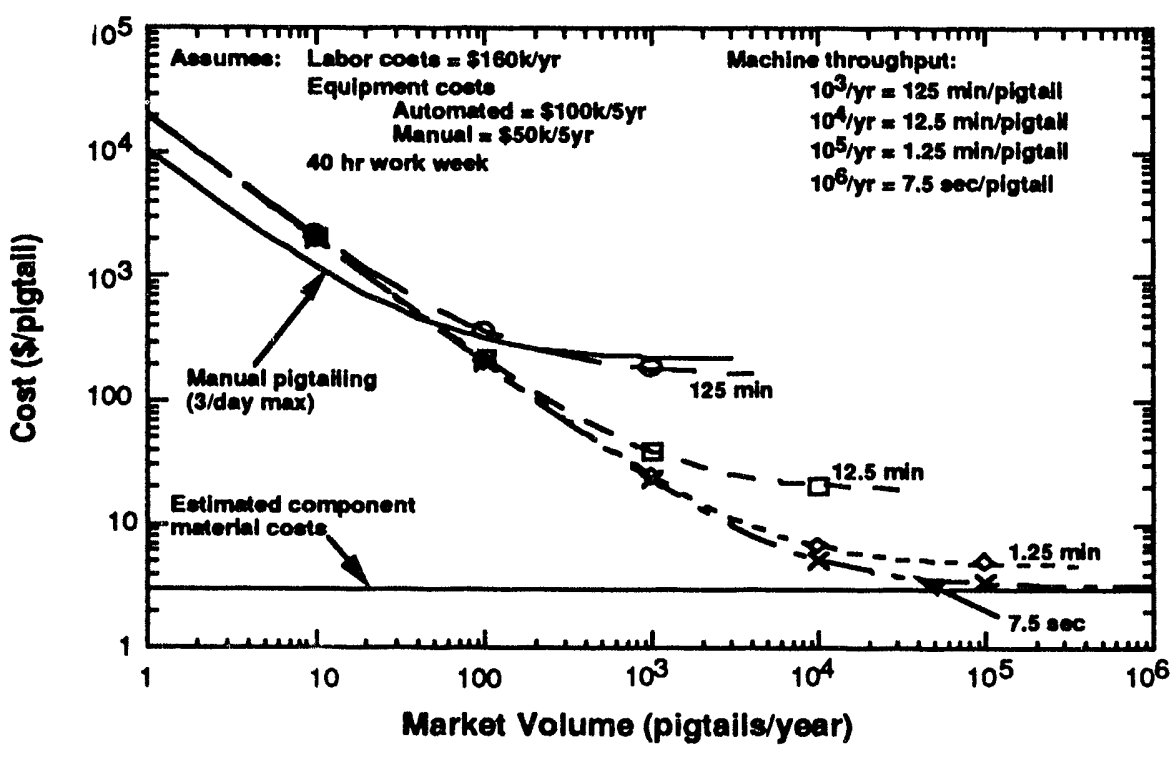

Figure 1. Substantial cost savings may be realized at even modest production rates. An automated system becomes less expensive than manual pigtailing at approximately 100 pigtails per year, or one pigtail every two or three days. aging process must be automated to significantly reduce the costs of OE devices. The electronics industry has successfully reduced the costs of its products through the massive use of automation, including robotics, parts handling and feeding, and in-situ quality control. A simple model (Figure 1), which takes into account the initial cost of the automated machinery, the labor costs of an operator, and the material costs of the devices, shows that substantial cost savings may also be realized in the optoelectronic industry at even modest production rates. Unfortunately, the sub-micron precisions required for OE packaging greatly exceed the requirements of the electronics industry. The automated systems developed to assemble integrated circuits cannot be applied to the problem of packaging optoelectronic circuits. 


\section{AUTOMATED FIBER ALIGNMENT}

\section{Our AFPM achieves sub-micron accuracies}

For the past several years, we have been working on a project to design and build a machine for automating the packaging of $\mathrm{OE}$ devices. The initial task of our Automated Fiber Pigtailing Machine (AFPM) will be to align a single-mode fiber to each end of a Mach-Zehnder modulator. The AFPM (Figure 2) uses a two-step alignment procedure to achieve sub-micron accuracies. The first step of the alignment, the so-called coarse alignment, uses a two-view camera system and object-recognition software to identify and locate the optical fiber and the waveguides in the lithium niobate substrate. The computer then sends the commands to move the fiber within a few microns of the waveguide; this is sufficiently accurate to couple some light between the waveguide and the fiber. For the second step of the alignment, which we call the fine alignment, a laser diode is turned on and the light passing between the components is monitored with a photodiode while the sub-micron stages complete the alignment. The fine adjustments are made by maximizing the amount of light coupled between the fiber and the modulator This combination of coarse and fine positioning techniques will result in faster, less expensive automated systems.

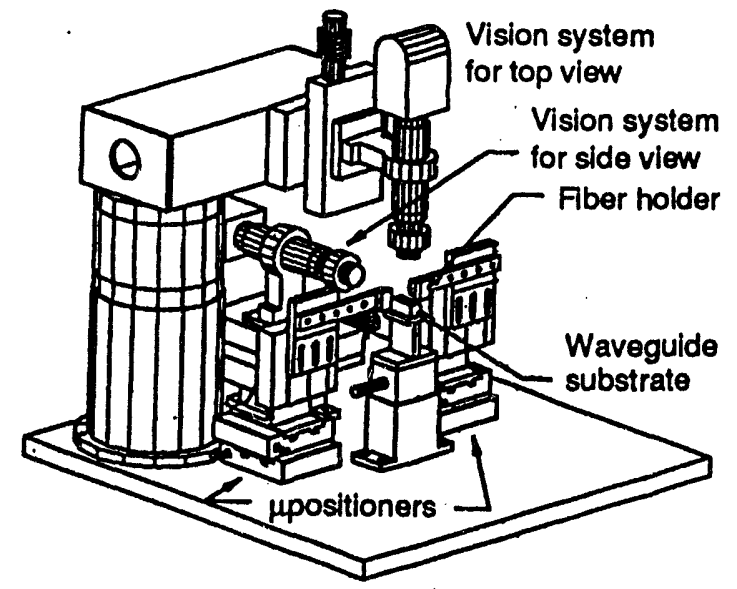

Figure 2. Our AFPM uses a two-step alignment procedure to obtain sub-micron accuracy.

\section{Sub-micron alignment takes only a few minutes}

The software that controls the AFPM and analyzes the camera images is presently in C-language on a 486-based PC; we are planning to make the software compatible with a Macintosh, also. The views of the top and side CCD cameras are recorded by a framegrabber with $480 \times 640$ pixels which provides approximately 800 micron fields-of-view with approximately $1.4 \mathrm{mi}$ cron per pixel resolution. Both cameras are mounted on translation stages to provide automatic zoom and focus as well as to view different areas on the modulator; in addition, the top camera is mounted on a rotary stage to move that camera out of the way for the application of epoxy to attach the fibers. The stages supporting the camera systems are high precision stages; the positions of the cameras are repeatible within 2 to 5 microns. There are some alignment procedures in which the objects required to perform a given alignment cannot all be contained within a single field-of-view. It is often necessary to accurately move from one field-of-view to another along the modulator or to move between two focal planes without losing absolute position information. The stages that position the fibers are Newport/Klinger PM500 with $25 \mathrm{~mm}$ of travel and 0.05 micron resolution. These stages have sufficient travel and sufficient resolution to satisfy the requirements for both the coarse and fine alignment procedures. At present, the computer takes approximately 10 seconds to acquire and analyze the images from the cameras; the subsequent coarse alignment motion of the stages takes less than 1 second. Generally, 6 to 10 images are needed to move the fiber from outside the camera field of view to within a few microns of the modulator waveguide. This means that the coarse alignment takes one to two minutes. The fine alignment of maximizing the amount of light passing between the components uses the patented AutoAlign "hill-climbing" algorithms developed by Newport/ Klinger. This procedure also takes one to two minutes for a total time to perform sub-micron alignments of less than five minutes. Future hardware and software upgrades should reduce this time by nearly an order of magnitude.

\section{The AFPM could be used for passive alignment}

The active alignment procedure being applied to the modulator in this prototype AFPM is also directly applicable to pigtailing laser diodes; the laser diode itself could be turned on to provide the light output which would be monitored at the opposite end of the fiber being pigtailed. There are many applications, however, in which passive components require high precision alignments. We are investigating the use of tactile feedback to perform the ine alignment of, say, flip-chip bonded components or fibers into silicon V-grooves. In this scenario, the vision system would be used to align the component to within a few microns, as before, and then the fine alignment system would achieve the highprecision alignment by monitoring a tactile feedback system. Multi-mode fiber attachments generally do not require such high precisions, so the AFPM could perform those alignments using only the coarse alignment vision system.

\section{AUTOMATED FIBER ATTACHMENT}

\section{We are developing attachment techniques compatible with automation}

Full advantage of the short alignment times achieved by the AFPM cannot be realized unless all other processes associated with the packaging are also short in duration. An important ad- 
ditional aspect of our automated packaging concept is the pigtailing of the optical fiber to the OE device. We are designing and building silicon microbenches with geometries that are compatible with automated processes. Silicon microbenches provide the stability required to maintain the sub-micron alignment tolerances necessary for single mode operation.

\section{Our silicon microbenches have on-boand heaters for fiber attachment}

We design our microbenches with discrete areas for $O E$ device attachment and areas for fiber attachment. For example, the microbench shown in Figure 3 is for packaging a $1550 \mathrm{~nm}$ DFB laser. This microbench is $13 \mathrm{~mm}$ long by $6 \mathrm{~mm}$ wide and 0.5 $\mathrm{mm}$ thick. On the left half of the microbench, we photolitho- ers to pigtail single-mode fibers to high-powered $800 \mathrm{~nm}$ laser diodes (Figure 4). The performance of the polysilicon heaters on this prototype is very reproducible using a power supply that allows us to accurately control the magnitude and time of the applied current. For this application, we use active feedback to align the fiber to sub-micron accuracies. While the fiber is held in the position that maximizes the optical coupling, current is passed through the heater to reflow the solder; the solder then wicks around the metalized fiber. We typically apply one amp of current for approximately $0.5 \mathrm{sec}$ to reflow the solder We observe no decrease in the light coupled from the $800 \mathrm{~nm}$ laser diode into the single-mode fiber after the solder has cooled and have achieved up to $65 \%$ optical coupling efficiency with conically tapered fibers.

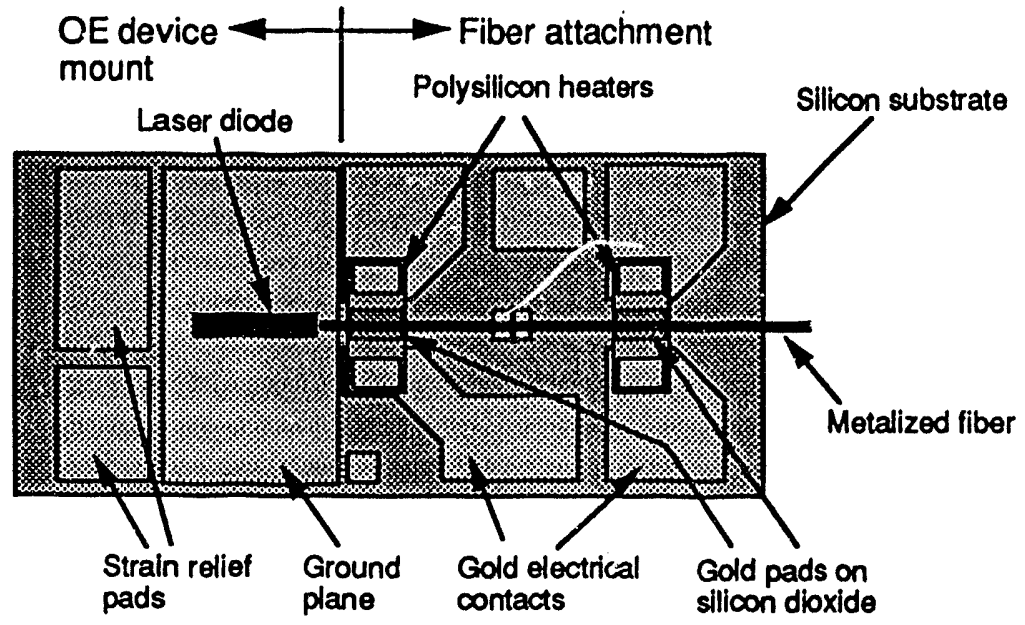

Figure 3. Our silicon microbenches use on-board polysilicon heaters to simplify component attachment and fiber pigtailing.

graphically pattern gold pads to provide a ground plane for the laser and stress relief pads for the wire bonds. To attach the fiber on the right half of the microbench, we pattern two heating elements made of polysilicon which are connected to gold bonding pads for electrical contact. In the center of each heater we pattern a gold pad on a layer of silicon dioxide. This gold pad provides the solder attachment base while the silicon dioxide electrically isolates the gold pad from the polysilicon heater The gold pads are $1 \mathrm{~mm}$ by $0.5 \mathrm{~mm}$ each and are suficiently large to solder a 125 micron diameter fiber at each attachment pad. Presently, we use either 100-micron diameter solder balls or solder paste to attach the metalized fiber. These on-board heaters eliminate the need for cumbersome soldering irons or hydrogen flame set-ups to reflow the solder.

\section{The on-board heaters allow sub-micron alignment of single mode fibers}

We originally built silicon microbenches with on-board heat-

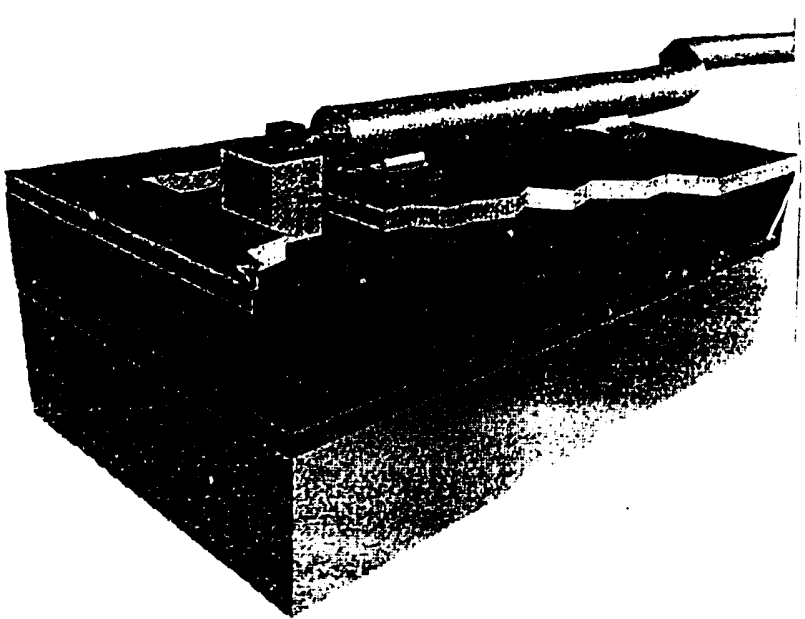

Figure 4. The polysilicon on-board heaters are compatible with sub-micron alignment tolerances.

\section{On-board heaters simplify the attachment of other components}

Our microbench geometries with on-board heaters allow rapid attachment of not only the fiber but also other components to be placed on the microbench. Applying larger currents for longer periods of time allows solder reflow at other locations on the microbench. Using solders with different melting temperatures and judiciously choosing the order of attachment allows a variety of components to be soldered to the microbench without movement of previously attached components. Generally components furthest from a heater are attached first using a high cur rent through the heater. We can hand solder a thermo-electric cooler, a thermistor, and a laser diode onto our microbench at different distances from the heaters in less than 15 minutes. The placement of these components does not require sub-micron alignment; we envision that the placement and soldering of these components onto the microbenches could be performed by an automated system in only a few seconds. As the last step, the fiber 
must be aligned to sub-micron accuracies and then be attached using the least current through the heaters.

We are designing microbench geometries for other applications

The idea of on-board heaters lends itself to applications other than packaging laser diodes. We are presently designing a longer microbench with heaters at each end to pigtail both ends of a semiconductor optical amplifier As hybrid OEMCM geometries become more complex, on-board heaters could provide more flexibility in the packaging process. On-board heaters could be designed to achieve solder reflow for bump-bond attachment; this would provide localized heating rather than elevating the temperature of the entire substrate.

\section{CONCLUSION}

The key element in reducing the costs of packaged optoelectronic devices is to minimize the manual labor costs. According to our model, manual p: jtailing techniques will not allow the cost of a pigtailed OE device to drop below approximately $\$ 250$. On the other hand, an automated process could allow the costs to drop as low as $\$ 10$ per pigtail-a factor of 25 decrease in cost. A fully automated system for fiber pigtailing must include automated fiber alignment, fiber attachment techniques which are compatible with an automated process, in-situ quality control, and automated parts handling and feeding. We present here our efforts in addressing the alignment issue and a fiber attachment technique. Variations of an automated system such as this could perform not only sub-micron active alignments as we discuss here, but also passive alignments using tactile feedback rather than optical feedback. The important point is to automate the process regardless of the alignment technique involved. We believe that a massive market is ready for the technology that optoelectronics can provide and is waiting for the costs to be reduced.

\section{ACKNOWLEDGEMENTS}

This work was performed under the auspices of the US Department of Energy by Lawrence Livermore National Laboratory under contract No. W-7405-Eng-48. 

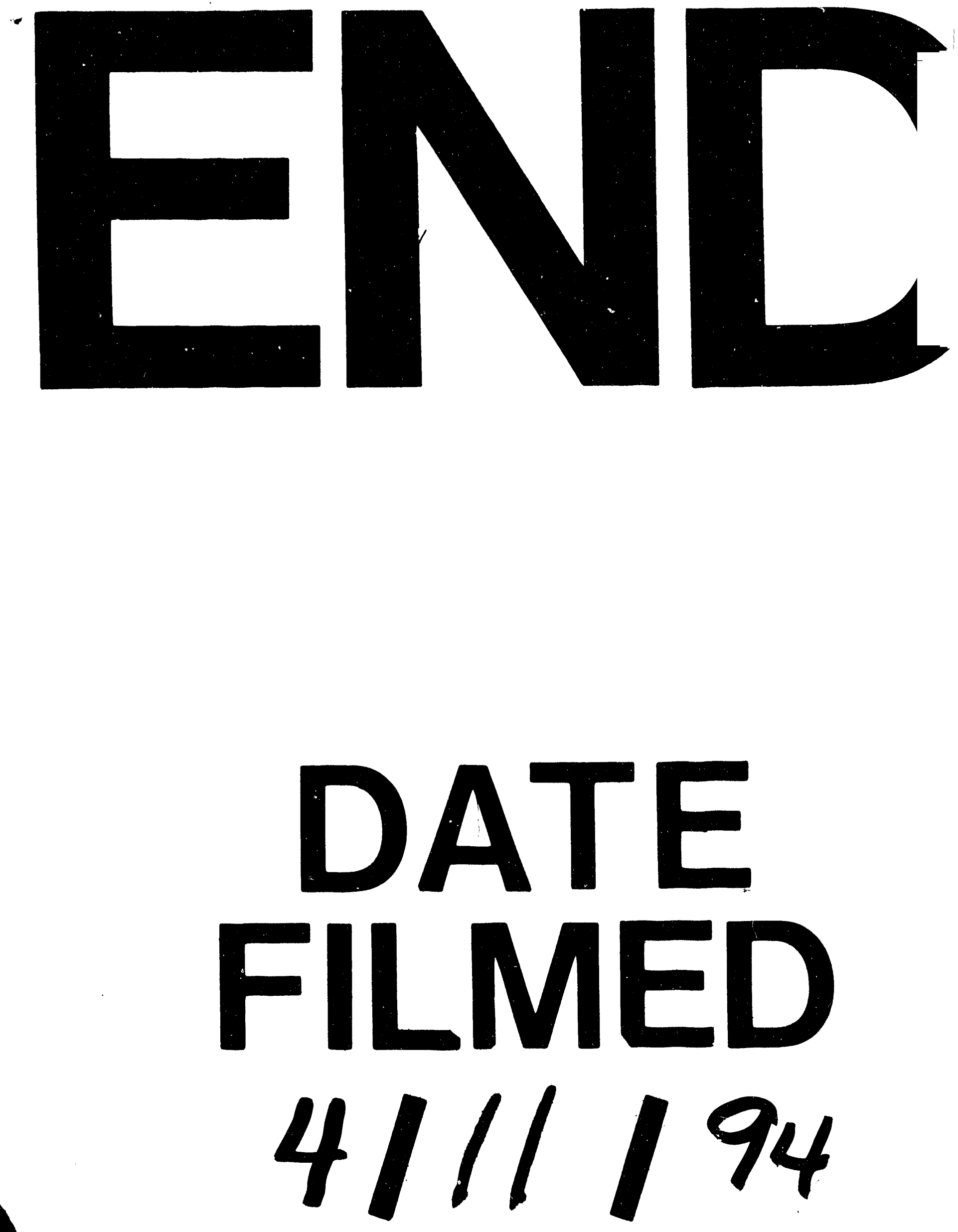
